\title{
Chirality Affecting Reaction Dynamics of HgS Nanostructures Simultaneously Visualized in Real and Reciprocal Space
}

\section{Zetan Cao}

Shanghai Jiao Tong University

Jia He

Shanghai Jiao Tong University

Zhiwen Liu

Shanghai Jiao Tong University

Haoran Zhang

Shanghai Jiao Tong University

Bin Chen ( $\nabla$ cbcce@sjtu.edu.cn )

Shanghai Jiao Tong University

\section{Article}

Keywords: redox reactions, chirality, structural dynamics, inorganic chiral nanostructures, in-situ observation

Posted Date: February 8th, 2021

DOl: https://doi.org/10.21203/rs.3.rs-142756/v1

License: (c) (1) This work is licensed under a Creative Commons Attribution 4.0 International License. Read Full License 


\title{
Chirality Affecting Reaction Dynamics of HgS Nanostructures Simultaneously Visualized in Real and Reciprocal Space
}

\author{
Zetan Cao, Jia He, Zhiwen Liu, Haoran Zhang, Bin Chen *
}

Center for Ultrafast Science and Technology, School of Chemistry and Chemical Engineering, and School of Physics and Astronomy, Shanghai Jiao Tong University, Shanghai 200240, China

E-mail: cbcce@sjtu.edu.cn 


\begin{abstract}
:
Chirality involved reactions enable to probe features in the fields of asymmetric synthesis and catalysis, which allow to gain insight into the fundamental mechanisms of topochemically controlled reactions. However, in situ observation of the chirality-associated reaction dynamics with simultaneous structural determination of new features has been lacking. Here, we report the direct visualization of the electronbeam-stimulated reaction dynamics of $\mathrm{HgS}$ nanostructures with chiral and achiral morphologies simultaneously in both real and reciprocal space. Under the electron-beam excitation of $\mathrm{HgS}$ nanostructures, the formation and evaporation dynamics of $\mathrm{Hg}$ nanodroplets were vividly pictured while the reciprocal space imaging revealed the structural transformation from monocrystalline to polycrystalline. Such induced changes were size-dependent, which were slowed down when involving the chirality in the nanostructures. The finding offers a fundamental understanding of topochemically controlled reaction mechanisms and holds promise of tuning asymmetric synthesis for catalysis related applications.
\end{abstract}

KEYWORDS: redox reactions; chirality; structural dynamics; inorganic chiral nanostructures; in-situ observation 
Chirality is usually used to describe the geometric property of handedness that is not superimposable on its mirror image by any combination of translations and rotations. Chiral molecules exist in two stereoisomers (enantiomers) that are mirror images of each other, showing either lefthanded or right-handed form by their configuration. Generally, both the left- and right-handed forms present similar chemical and physical properties, except when they are utilized in particular cases including asymmetric catalysis ${ }^{1,2}$, optical activities ${ }^{3}$ and biological processes ${ }^{4-6}$. Chirality occurs commonly in organic and biological molecules/compounds ${ }^{7-10}$. Nevertheless, inorganic chiral entities possess distinctive characteristics such as the facile control of crystal structures and the tolerance to high-temperature stresses, which exhibit great promise for applications in chiral sensing ${ }^{11-13}$, advanced optical devices $^{14-21}$, and asymmetric catalysis ${ }^{22-25}$.

Among the 230 types of space groups inorganic chiral nanocrystals belong to the 65 Sohncke space groups ${ }^{26,27}$, where the group is non-superimposable with its mirror image enantiomorph. Chirality in these systems exists at all length scales, from the atomic/molecular level (lattice chirality), up to the macroscopic scale (morphology chirality). Inspired by the chirality in nature and life, most studies of inorganic chiral nanocrystals have been devoted to the synthesis strategies in the past years ${ }^{28-}$ 36. Typical applications of chiral crystals, promising in optical devices and photocatalysis, require the involvement of the interaction with photons/electrons, such as irradiation. However, the reports of chiral effect on the irradiation dynamics are rare.

$\mathrm{HgS}$, chiefly used as a pigment in paints throughout history, occurs as a red (cinnabar, $\alpha-\mathrm{HgS}$, hexagonal) or black (metacinnabar, $\beta-\mathrm{HgS}$, cubic) crystalline solid. $\alpha-\mathrm{HgS}$ belongs to the chiral space group $P 3121$ or $P 3_{2} 21$, showing both the lattice and morphology chirality ${ }^{34}$ that would be ideal for studying the irradiation-induced chemical reaction dynamics. Under pulsed laser ablation with the 
wavelengths from ultraviolet to near infrared, the phase transformation from $\alpha$ - to $\beta$-HgS was observed by the micro-Raman spectroscopy and X-ray photoelectron spectroscopy methods ${ }^{37}$. Using the pumpprobe microscopy, both the $\beta-\mathrm{HgS}$ and metallic liquid $\mathrm{Hg}$ were created by the ultraviolet light exposure of $\alpha-\mathrm{HgS}^{38}$. However, in situ observation of such irradiation-induced chemical reaction process as well as its subsequent structural evolution in space and time has been lacking. Involving the chiral effect on the e-beam irradiation stability have been reported in multiwalled carbon ${ }^{39}$ and boron nitride ${ }^{40}$ nanotubes. Revealing simultaneously the chemical reaction dynamics in both real and reciprocal space, especially when involving chiral effect, is essential to provide an unambiguous understanding of chirality mediated reactions and phase transitions, which paves the way for controlling the chiralityassociated plasmonics and catalytic reactions.

Here, we report the direct observation of chirality-related chemical reaction dynamics of inorganic $\mathrm{HgS}$ nanostructures by using in situ transmission electron microscopy (TEM) simultaneously in real and reciprocal space. The technique enables the visualization of the formation and evaporation dynamics of liquid $\mathrm{Hg}$ nanodroplets as a result of the e-beam-stimulated chemical reduction reactions of $\mathrm{Hg}^{2+}$ to $\mathrm{Hg}^{0}$, and allows the simultaneous determination of the structural transition from monocrystalline to polycrystalline. Interestingly and surprisingly, the $\mathrm{HgS}$ nanostructures, once involving chirality, present distinct reaction behavior under the e-beam excitation. The chiral nanostructures show dense surface steps, which are more tolerant against the e-beam irradiation than that of the achiral ones. Such e-beam-stimulated reaction behavior is size-dependent, namely, the tolerance reaches the highest as the size of the nanostructure is around $200 \mathrm{~nm}$ and then becomes weakened as the size increases. The chiral effect on the above phenomena is discussed. 


\section{Results}

Synthesis and characterization of HgS nanostructures. The inorganic HgS nanostructures were synthesized using the seed-mediated epitaxial method with the growth rate controlled by a syringe pump (Fig. 1, top; see Methods, the left corner is an example of the as-synthesized product). The HgS seeds had a size of $\sim 8-15 \mathrm{~nm}$ (Supplementary Fig. 2), which were used for the subsequent epitaxial growth of various nanostructures. The nanostructures can crystallize in the chiral space group P3 21 or its enantiomorph P3221. Shown in Fig. 1 (middle left) is the atomic model of HgS with the P3121 space group ${ }^{41}$. The helical arrangement of $\mathrm{Hg}$ and $\mathrm{S}$ atoms along the crystallographic $c$ axis represents an atomic scale primary chiral unit (Fig. 1, middle center). The chiral unit acts as a template for the epitaxial growth of chiral $\mathrm{HgS}$ nanostructures in the presence of chiral molecules (Fig. 1, middle right). Fig. 1 (bottom left) shows a typical TEM image of the as-synthesized $\mathrm{HgS}$ nanostructures. The $\mathrm{HgS}$ displays twisted bipyramid morphology with the geometric left-handedness ${ }^{34}$, which has a width and length of $\sim 150,400 \mathrm{~nm}$ in size, respectively. Detailed growth conditions of $\mathrm{HgS}$ with different sizes are listed in Supplementary Table 2 and the corresponding morphologies are presented in Supplementary Fig. 3. The energy dispersive X-ray (EDX) spectrum and mapping results indicate the existence of the $\mathrm{Hg}$ and $\mathrm{S}$ elements in the nanostructure, and show no obvious aggregation of clusters before long-term electron beam (e-beam) illumination (Fig. 1, bottom center and right).

Experimental implementation of reaction dynamics studies. We investigated the chemical reaction dynamics of the $\mathrm{HgS}$ nanostructures with chiral and achiral morphologies in both real and reciprocal space using the in situ TEM (Fig. 2, left). An e-beam is employed to excite the specimen so that chemical reactions are triggered at targeted locations. The subsequent reaction dynamics are recorded simultaneously in real (structural imaging) and reciprocal (phase determination) space by a digital 
camera. Fig. 2 (bottom right) shows the morphologies of the chiral $\mathrm{HgS}$ before $(0 \mathrm{~s})$ and after $300 \mathrm{~s}$ ebeam irradiation, respectively. In comparison with the initial one, the nanostructure after the irradiation presents significant changes of contrast and the occurrence of many dispersed nanoclusters with dark contrast (marked by the red arrow).

High resolution TEM image reveals that the nanostructure after the e-beam irradiation was still $\alpha-\mathrm{HgS}$ phase, without the transformation to $\beta$ phase (Supplementary Fig. 4), which is different from that observed in the samples after the laser exposure ${ }^{37}$. It indicates that the behavior in this work might be associated with the chemical reactions. Because chemical species exhibit quite different mass loss rate, the overall chemical composition of the sample may change during the e-beam irradiation. Upon comparison of the EDX mapping results between the two states ( 0 and $300 \mathrm{~s})$, the losses of both $\mathrm{Hg}$ and S were clearly observed after the irradiation (Supplementary Fig. 5). The EDX spectra show that the intensities from both $\mathrm{Hg}$ and $\mathrm{S}$ elements decreased, where the ratio of $\mathrm{Hg}: \mathrm{S}$ quantitatively changed from $\sim 1: 1$ of the initial stoichiometric sample to $\sim 1: 2$ of the e-beam-irradiated one (Fig. 2, upper right). To understand the above phenomena, the reaction dynamical processes were in situ revealed in the following sections.

Reaction dynamics of chiral HgS nanobipyramids. The structural evolution dynamics of a single chiral $\mathrm{HgS}$ nanobipyramid were investigated during the e-beam exposure, as shown in Fig. 3 (Supplementary Movie 1). The nanobipyramid is $\sim 60,120 \mathrm{~nm}$ in width and length, respectively. For convenience, we neglected the time of the TEM alignment, and denoted the initial image as time zero $(0 \mathrm{~s})$. The image and its live fast Fourier transform (FFT) pattern indicate that the as-synthesized $\mathrm{HgS}$ is monocrystalline, corresponding to the trigonal structure $(a=4.145 \AA, b=4.145 \AA \text { and } c=9.496 \AA)^{42}$. The direction along the length of the nanobipyramid is parallel to the [001] orientation. After the e- 
beam excitation for $50 \mathrm{~s}$, several $\mathrm{Hg}$ nanodroplets $(\sim 8 \mathrm{~nm}$ in size) were seen (marked by the dotted circles). The occurrence of the $\mathrm{Hg}$ nanodroplets might be due to the e-beam-induced chemical reaction of HgS. The nanodroplets gradually decreased their size during the e-beam irradiation, and finally vanished in about $31 \mathrm{~s}$ (Supplementary Fig. 6). With the prolonged irradiation (120 and $180 \mathrm{~s}$ ), the nanovoids gradually grew and coalesced into the big ones. In comparison with the initial state, the diffraction spots became less and the intensity was weaker, indicating a lower symmetry of the $\mathrm{HgS}$ nanostructure. As the e-beam irradiation continued $(240 \mathrm{~s})$, the $\mathrm{HgS}$ nanostructure was severely destroyed and only several diffraction spots were still visible. Eventually, a hollow nanostructure with the twisting shell layer remained on the supporting film after the $300 \mathrm{~s}$ e-beam irradiation.

Size-dependent effect on reaction dynamics of chiral $\mathrm{HgS}$ nanobipyramids. To understand the formation of the $\mathrm{Hg}$ nanodroplets, we further studied the $\mathrm{HgS}$ nanobipyramids with different sizes under the e-beam excitation. Fig. 4 shows the tendency of the stability of $\mathrm{HgS}$ nanobipyramids with respect to their sizes (length ranged from 80 to $400 \mathrm{~nm}$; an example is displayed in the inset) under the same e-beam irradiation condition. The period from the initial state to the one that the morphology and corresponding FFT pattern of the nanostructure without change obviously during the irradiation was referred to the total reaction time in the figure. With increasing the size of the nanobipyramid, the total reaction time increased to $\sim 1400 \mathrm{~s}$ for the nanobipyramid with the size of $\sim 200 \mathrm{~nm}$, and then decreased to $\sim 400 \mathrm{~s}$ for the $\sim 400 \mathrm{~nm}$ one (Fig. 4, top). Four typical HgS nanobipyramids with the different sizes $(\sim 120,200,240$ and $340 \mathrm{~nm})$ are displayed in the bottom panel of the figure, corresponding to the dotted circles marked in the top panel. Under the identical e-beam irradiation for $240 \mathrm{~s}$, no obvious changes of the morphology and the corresponding FFT pattern were observed in the $\sim 200 \mathrm{~nm}$ nanobipyramid. In contrast, significant changes were seen in the nanobipyramids with other sizes. For 
example, the hollow nanostructure was already formed in the $\sim 120 \mathrm{~nm}$ nanobipyramid. For the large ones, a number of nanovoids appeared in the nanobipyramids with the sizes of 240 and $340 \mathrm{~nm}$. It suggests that the $\sim 200 \mathrm{~nm} \mathrm{HgS}$ nanobipyramid is more persistent to the e-beam irradiation than that with other sizes, resulting in the distinct chemical reaction rates observed above. It is noteworthy from the FFT pattern that the $\sim 340 \mathrm{~nm}$ nanobipyramid presents obvious transformation from monocrystalline to polycrystalline after the irradiation for the same time. For details, the reaction characteristics of $\mathrm{HgS}$ with other sizes were displayed in Supplementary Fig. 7-16.

Reaction dynamics of achiral HgS nanobipyramid. To further understand the chiral effect on the reaction dynamics, the e-beam irradiation of the achiral nanostructures was performed for the comparison. Fig. 5 shows the structural evolution of $\mathrm{HgS}$ nanobipyramid with chiral (top left) and achiral (top right) morphologies under the same e-beam excitation condition. The initial nanobipyramid is approximately $280 \mathrm{~nm}$ in size. When the irradiation time increased, the chiral $\mathrm{HgS}$ showed no obvious morphological changes and the diffraction spots with monocrystalline form were readily seen (0-20 s). However, significant changes were observed in the achiral $\mathrm{HgS}$, i.e., several $\mathrm{Hg}$ nanodroplets with the range of 3-8 $\mathrm{nm}$ in diameter were seen (marked by the dotted circles) after $20 \mathrm{~s}$ e-beam irradiation. It indicates that the achiral $\mathrm{HgS}$ nanostructures were less tolerance to the e-beam irradiation, in opposite to that observed in the chiral cases. Note that the evaporation time of the $\mathrm{Hg}$ nanodroplets for the achiral nanobipyramid was about $12 \mathrm{~s}$ (Supplementary Fig. 17), which was shorter than that for the chiral one $(\sim 31 \mathrm{~s})$. The atomic force microscopy (AFM) results show that the surface of the chiral nanobipyramid is rougher than that of the achiral one (Fig. 5, bottom). Moreover, the surface step heights of the chiral and achiral nanobipyramids are estimated to be $\sim 10$ and $20 \mathrm{~nm}$, respectively, indicating the denser surface steps on the chiral samples (Supplementary Fig. 18). It 
suggests that the chirality-affected chemical reaction dynamics might be associated with the surface characteristics of the nanostructures.

\section{Discussion}

Generally, sample damage caused by the e-beam can be through either elastic scattering such as knockon atomic displacements or inelastic scattering including specimen heating and radiolysis. It has been reported that continuous ultraviolet light exposure can generate $\mathrm{Hg}$ droplets through local heating of $\mathrm{HgS}^{38}$. Besides, for pure $\alpha-\mathrm{HgS}$ sample, the phase transformation to $\beta$-HgS occurs at $\sim 673 \mathrm{~K}$ and is completed at $\sim 698 \mathrm{~K}^{43}$. Under the e-beam irradiation shown here, however, the e-beam-induced temperature rise is no more than $1 \mathrm{~K}^{44}$ (see detailed discussion in Supplementary Information including Supplementary Fig. 1 and Table 1). This suggests that heating is impossible to cause phase transformation and has negligible effect on the formation of $\mathrm{Hg}$ nanodroplets.

It is seen from Figure 2 (top right) that the loss of $\mathrm{Hg}$ (higher atomic mass) is faster than that of $\mathrm{S}$ after the e-beam irradiation, suggesting that the knock-on mechanism is not the major factor for the sample damage. With the exposure to high-energy e-beam, the ionizing radiation (radiolysis) would cause the breakage of chemical bonds in materials. Based on these considerations and the experimental results, a pictorial understanding of the e-beam-induced chemical reactions in $\mathrm{HgS}$ system is illustrated in Fig. 6 (top). Under the e-beam stimulus, the cleavage of $\mathrm{Hg}$-S chemical bonds and the creation of vacancies readily occur, which may induce the reactions of the reduction of $\mathrm{Hg}^{2+}$ to $\mathrm{Hg}^{0}$ and the oxidization of $\mathrm{S}^{2-}$ to $\mathrm{S}^{0}$. The $\mathrm{Hg}^{0}$ atoms diffuse and aggregate so that the $\mathrm{Hg}$ nanodroplets nucleate and grow. As seen from the FFT patterns (Supplementary Fig. 7-16.), a lower symmetry of the $\mathrm{HgS}$ nanostructure appears at this stage (Supplementary Movie 2). Owing to the subsequent evaporation of 
many $\mathrm{Hg}$ nanodroplets, a number of nanovoids emerge, which divide the original single crystal into many pieces of small polycrystals. As the Hg nanodroplets continue to move and evaporate, the nanosized polycrystals would gradually rotate and change their orientations, giving rise to the FFT ring patterns (structural transformation from monocrystalline to polycrystalline) observed in the later stage of the e-beam irradiation. Finally, the nanostructures are disordered during the long-term e-beam irradiation, resulting in the amorphization of the most parts of the specimen.

In general, the tolerance against the e-beam irradiation increase/decrease linearly with respect to the size of nanostructured materials ${ }^{45-47}$. The size-dependent chemical reaction dynamics observed in this work, different from general cases, may involve the interplay between the surface energy of steps and the free energy of irradiation-induced defects (e.g., vacancies). During the e-beam bombardment, such generated point defects would diffuse and be absorbed to the surface steps of $\mathrm{HgS}$ nanobipyramid, and finally annihilate there. The excess free energy $\Delta G_{d}$ of these point defects is proportional to the square of nanobipyramid size $L$, i.e., $\Delta G_{d} \propto L^{2}$, while the free energy change of surface steps $\Delta G_{s}$ is described as $\Delta G_{S} \propto 1 / L^{48}$. Thus, the change in Gibbs free energy of the irradiated HgS nanobipyramid is the sum $\Delta G_{\text {sum }}$ of the two, namely, $\Delta G_{s}+\Delta G_{d}$, as shown in Fig. 6 (bottom left). When the crystal size is in the range of $L<L_{1}$ or $L>L_{2}$, the Gibbs free energy barrier of the transition satisfies $\Delta G_{t}<\Delta G_{\text {sum }}$, indicating that the transition (a change in crystal structure or amorphization) would be easily induced by the e-beam irradiation. Our experimental results of $\mathrm{HgS}$ nanobipyramids with the sizes of $80 \sim 160 \mathrm{~nm}$ or $240 \sim 400 \mathrm{~nm}$ follow the criteria in this region. On the other hand, it becomes harder for the transition when $L_{1}<L<L_{2}$ since $\Delta G_{t}>\Delta G_{\text {sum }}$. As observed in Fig. 4, the high irradiation tolerance occurred in the $\mathrm{HgS}$ nanobipyramids with the sizes of 180 220 nm provides the information of size-dependent stability in $\mathrm{HgS}$ nanostructured system during the irradiation. 
Chirality has a significant role in the surface morphology of $\mathrm{HgS}$ nanobipyramids, resulting in the distinct dynamics observed during the e-beam stimulus (Fig. 5). Upon comparison of the phenomena in the chiral and achiral $\mathrm{HgS}$, it suggests that the chiral morphology with high density of surface steps is more resistant to the e-beam irradiation than the achiral one. The great quantity of the surface steps would function as the sinks for the removal of irradiation-induced defects. Meanwhile, the wetting process on the nanobipyramid surface would determine the nucleation of $\mathrm{Hg}$ nanodroplets as a result of the chemical reactions induced by the e-beam excitation. The formation of $\mathrm{Hg}$ nanodroplets depends on the interplay of several contributions (Fig. 6, bottom center) to the Gibbs free energy that are expressed $\mathrm{as}^{49}$,

$$
\begin{gathered}
\Delta G=G_{v o l}+G_{\text {surface }} \\
G_{v o l}=g_{v o l} V
\end{gathered}
$$

where $G_{v o l}$ is the volume free condensation energy, $G_{\text {surface }}$ the surface energy, $g_{v o l}$ the free energy per unit volume for $\mathrm{Hg}\left(-13.43 \mathrm{eV} / \mathrm{nm}^{3}\right)^{50,51}$ and $V$ the volume of $\mathrm{Hg}$ nanodroplet. The surface energy $G_{\text {surface }}$ involves the contributions from the surface tensions and the contact areas of solidliquid and liquid-vacuum interfaces. According to the Young's equation, the interfacial tensions are expressed with the contact angle as,

$$
\cos \theta=\left(\gamma_{s v}-\gamma_{s l}\right) / \gamma_{l v}
$$

where $\gamma_{l v}, \gamma_{s l}$ and $\gamma_{s v}$ are the interfacial tensions of liquid-vacuum, solid-liquid and solid-vacuum, respectively. The exact value of the volume free condensation energy and surface energy depend on the contact angle $\theta$. Consequently, the size of the critical nucleus $R^{*}$ could be determined by $G_{v o l}$ and $G_{\text {surface }}$ energies, which is varied with the change of contact angle $\theta$ (Fig. 6, bottom right). Assuming the classical cap shape for the nanodroplets, the expressions of the critical radius and the 
critical total free energy change $\Delta G^{*}$ for their heterogeneous nucleation are described as follows (see detailed calculations in Supplementary Information),

$$
\begin{gathered}
R^{*}=-\frac{2 \gamma_{l v}}{g_{v o l}} \sin \theta \\
\Delta G^{*}=\frac{4 \pi \beta \gamma_{l v}^{3}}{3\left(g_{v o l}\right)^{2}} \\
\beta=(2+\cos \theta)(1-\cos \theta)^{2} \\
J=J_{0} \exp \left(-\Delta G^{*} / k_{B} T\right)
\end{gathered}
$$

Substituting the interfacial tension $\gamma_{l v}$ value $(0.486 \mathrm{~N} / \mathrm{m})^{52}$ into eq. (4), the critical radius and total free energy for the $\mathrm{Hg}$ nanodroplet nucleation on the $\mathrm{HgS}$ surface was determined with respect to the contact angle $\theta$, as shown in Fig. 6 (bottom right) and Supplementary Fig. 6. The critical total free energy $\Delta G^{*}$ and nucleation rate $J$ for heterogeneous nucleation of $\mathrm{Hg}$ nanodroplets depend on the intrinsic wettability of the surface via the contact angle. The contact angle could be obtained from the evaporation dynamics of the $\mathrm{Hg}$ nanodroplets. The evaporation time $\tau$ is given by ${ }^{53}$

$$
\begin{gathered}
\tau=\frac{1}{4}\left(\frac{3}{\pi}\right)^{2 / 3} \frac{V_{L 0}^{2 / 3}}{D} \frac{k_{B} T \beta^{1 / 3}}{P_{0} V_{m} f} \frac{C_{f}}{e^{\lambda / R}-1+x} \\
P_{V}=(1-x) P_{0} \\
f=0.5\left(0.00008957+0.6333 \theta+0.116 \theta^{2}-0.08878 \theta^{3}+0.01033 \theta^{4}\right)
\end{gathered}
$$

where $J_{0}$ is the kinetic constant, $V_{L 0}$ the initial volume of the nanodroplet with a contact diameter $R$, $V_{m}$ the $\mathrm{Hg}$ atom volume in the liquid phase $\left(2.48 \times 10^{-2} \mathrm{~nm}^{3}\right), k_{B}$ the Boltzmann's constant, $T$ the temperature $\left(25^{0} \mathrm{C}\right), D$ the diffusion constant $\left(14.2 \times 10^{-6} \mathrm{~m}^{2} / \mathrm{s}\right)^{54}, P_{0}$ the saturated vapor pressure $\left(1.6 \times 10^{-4} \mathrm{~Pa}\right)^{55}, \theta$ the contact angle in radians, $\lambda$ the characteristic length scale for $\mathrm{Hg}$ liquid drops $(5.81 \mathrm{~nm}), C_{f}$ the factor describing the nanodroplet evaporation in the nanoscale ${ }^{56,57}, P_{V}$ the external vapor pressure. From eq. (9) the value of $x$ was calculated to be 0.99 by knowing that the external vapor pressure was $\sim 10^{-7} \mathrm{~Pa}$ inside the TEM chamber. From the total evaporation time of the $\mathrm{Hg}$ 
nanodroplets on the chiral ( $\sim 31$ s, Fig. 3 and Supplementary Fig. 6$)$ and achiral ( $\sim 12$ s, Supplementary Fig. 17) $\mathrm{HgS}$ surfaces, the contact angles were estimated from eq. (8-10) to be $\sim 126^{\circ}$ and $\sim 66^{\circ}$, respectively. At these contact angles, the total free energy of the $\mathrm{Hg}$ nanodroplet was $2.31 \mathrm{eV}$ for the chiral morphology while it decreased to $0.55 \mathrm{eV}$ for the achiral one. An estimate of the nucleation rate on a surface with contact angle of $\sim 66^{\circ}$ about $10^{29}$ times higher than that of $\sim 126^{\circ}$ surface from eq. (7). This indicates that it became easier for the $\mathrm{Hg}$ nanodroplet nucleation on the achiral $\mathrm{HgS}$ surface than that on the chiral one. Our experimental observations agree with the tendency reported in the water droplet system that the calculated nucleation rate on its surface with a contact angle of $\sim 25^{\circ}$ was considerably higher than that of $\sim 110^{\circ}$ surface $^{58}$. In the extreme case, when the contact angle is zero (condition for liquid spreading), the total free energy change $\Delta G$ equals zero, suggesting no energy barrier for the heterogeneous nucleation. Thus, it becomes relatively hard to trigger the chemical reactions in the chiral $\mathrm{HgS}$ nanostructures (large contact angles) under the e-beam stimulus, as observed experimentally in Fig. 5.

In conclusion, the real-time observations of the e-beam-stimulated reaction dynamics involved in the chiral and achiral $\mathrm{HgS}$ nanobipyramids, together with the simultaneous identification of their structural transformation, reveal the nature of size-dependent chiral effect on the nucleation and evaporation dynamics of the reaction-induced liquid nanodroplets. With such in situ real and reciprocal space visualization, we obtain the evaporation rate of the $\mathrm{Hg}$ nanodroplets for both the chiral and achiral system, facilitating the quantitative understanding of the chirality associated reaction dynamics of inorganic enantiomers in terms of the free energies accounting for the nucleation and evaporation processes. This approach has the potential for unique applications in chemistry and materials science, providing the key parameters accounting for the transient behaviour of topochemically controlled 
reactions at the nanoscale.

\section{Methods}

\section{Synthesis of $\alpha-H g S$ seeds}

In a solution-based synthesis, $70.0 \mathrm{mg}$ of $\mathrm{Hg}\left(\mathrm{NO}_{3}\right)_{2} \cdot \mathrm{H}_{2} \mathrm{O}$ and $10.0 \mathrm{~mL}$ of deionized water were mixed in a $25 \mathrm{~mL}$ glass vial, followed by injection of $2.0 \mathrm{~mL}$ of aqueous solution containing $26.9 \mathrm{mg}$ of D-penicillamine (or L-penicillamine or equal mixture of D- and L- penicillamine). Then, a $0.6 \mathrm{~mL}$ of aqueous solution containing $24.0 \mathrm{mg}$ of $\mathrm{NaOH}$ was added into the vial under magnetic stirring. With the dropwise addition of $2.0 \mathrm{~mL}$ of thioacetamide solution $(0.1 \mathrm{M})$, the color of the mixture solution turned from colorless to dark brown. The reaction was proceeded in a $38{ }^{\circ} \mathrm{C}$ water bath for 15 $\mathrm{h}$ under stirring. The product was collected by centrifugation at $6,000 \mathrm{rpm}$ for $10 \mathrm{~min}$, washed three times using isopropanol, and finally dispersed in $5.0 \mathrm{~mL}$ of deionized water.

\section{Seed mediated epitaxial growth of $\alpha-\mathrm{HgS}$ with chiral and achiral morphology}

The epitaxial growth of $\alpha-\mathrm{HgS}$ with chiral morphology was performed using a syringe pump at the specific growth temperature (Figure 1, top). In a typical synthesis, $50 u \mathrm{~L}$ of $\alpha$-HgS seeds colloidal solution and $5 \mathrm{~mL}$ water were added into a three-neck round-bottom flask submerged in the $38{ }^{\circ} \mathrm{C}$ water bath. $1.0 \mathrm{~mL}$ of aqueous solution containing $13.5 \mathrm{mg}$ of D-penicillamine (or L-penicillamine or equal mixture of $\mathrm{D}$ - and L-penicillamine) and $0.6 \mathrm{~mL}$ of aqueous solution containing $24.0 \mathrm{mg}$ of $\mathrm{NaOH}$ were successively added into the flask under magnetic stirring. Then, $6.0 \mathrm{~mL}$ of aqueous solution containing $74.4 \mathrm{mg}$ of $\mathrm{Hg}\left(\mathrm{NO}_{3}\right)_{2} \cdot \mathrm{H}_{2} \mathrm{O}$ ( $\mathrm{Hg}$ precursor solution) and $10 \mathrm{~mL}$ of aqueous solution containing $19 \mathrm{mg}$ of thioacetamide (S precursor solution) were co-injected into the flask through the syringe pump with an injection rate of $1.0 \mathrm{~mL} / \mathrm{h}$. To obtain the chiral $\alpha-\mathrm{HgS}$ with different sizes, the 
injection time was controlled from 2 to $6 \mathrm{~h}$. After reaction, the obtained colloidal solution (orange color) was centrifuged at $6,000 \mathrm{rpm}$. The precipitates were washed three times using isopropanol, and finally dispersed in $5.0 \mathrm{~mL}$ of deionized water.

\section{TEM sample preparation and characterization}

The TEM samples were prepared by drying the drops of the $\alpha-\mathrm{HgS}$ colloidal suspension on the carbon-coated copper grids under ambient conditions. To avoid the aggregation of the nanocrystals, the colloidal suspension was sonicated for about 10 minutes before dropping on the copper grids. The structural characterization of the samples were carried out using a ThermoFisher Talos F200X TEM with an accelerating voltage of $200 \mathrm{kV}$. The energy-dispersive X-ray detector (EDX) was used to conduct elemental analysis of the samples. As-synthesized $\mathrm{HgS}$ nanostructures were thoroughly characterized by atomic force microscope (AFM, FastScan Bio, Bruker, USA). AFM topography imaging and line profile analysis were used to reveal and measure the step height and thickness.

\section{In situ visualization of structural dynamics}

In situ observations of the e-beam-induced structural dynamics of $\alpha-\mathrm{HgS}$ nanocrystals were performed in the TEM with a constant e-beam dose rate of $700 \mathrm{e} / \AA^{2} \mathrm{~s}$. We recorded simultaneously the structural evolution of $\alpha-\mathrm{HgS}$ nanocrystals and their corresponding fast Fourier transform (FFT) patterns by a digital camera. All the movies for each kind of specimen were recorded at the same condition. The EDX and high-angle annular dark-field (HAADF) imaging were employed to analyze the composition changes of the $\alpha-\mathrm{HgS}$ nanocrystals at different e-beam irradiation time. 


\section{Acknowledgements}

We thank X. Guo for technical assistance. We thank the National Natural Science Foundation of China (No. 92061116) for the support. Financial supports from the Science and Technology Commission of Shanghai Municipality (20JC1416600), the Program for Professor of Special Appointment (Eastern Scholar) at Shanghai Institutions of Higher Learning, and Shanghai Pujiang Program (18PJ1405900) are acknowledged.

\section{Figure captions}

Figure 1. Synthesis and structural characterization of $\boldsymbol{\alpha}-\mathrm{HgS}$ nanobipyramids. Upper: Schematic of the seed-mediated epitaxial growth of chiral $\alpha-\mathrm{HgS}$ nanostructures. The inset shows the orange $\alpha$ $\mathrm{HgS}$ colloidal suspensions. Middle left: Schematic representation of the atomic structure of $\alpha-\mathrm{HgS}$ (Space group P3121). Middle center: Helical chains of $\mathrm{Hg}$ and $\mathrm{S}$ atoms along the crystallographic $c$ axis. Middle right: Schematic illustration of the epitaxial evolution from the chiral lattice to the chiral morphology by involving the chiral molecules. Bottom left: Typical TEM image of $\alpha$-HgS with chiral morphology. The dotted lines are the twisting orientations in the nanobipyramids. Bottom center: Elemental mapping of $\mathrm{Hg}$ and $\mathrm{S}$, respectively. Bottom right: EDX spectrum of of $\alpha-\mathrm{HgS}$. The inset is the corresponding high-angle annular dark field (HAADF) image for EDX.

Figure 2. Experimental setup for in-situ observations of chemical reaction dynamics. Left: Schematic of e-beam-induced chemical reaction dynamics visualized in both real and reciprocal space. The sample dynamics are triggered by an e-beam, while their structural evolution and corresponding diffraction patterns are simultaneously recorded by a charged-coupled device (CCD) camera. Upper 
right: EDX spectra of $\alpha-\mathrm{HgS}$ before and after the 300 s e-beam irradiation. Bottom right: TEM images of $\alpha$-HgS before and after the $300 \mathrm{~s}$ e-beam irradiation. The arrow indicates the occurrence of nanoclusters due to the irradiation.

Figure 3. Structural evolution in the chiral $\boldsymbol{\alpha}$-HgS nanobipyramid. Time-resolved TEM images and live FFT patterns show the formation and evaporation dynamics of $\mathrm{Hg}$ nanodroplets as well as the creation and coalescence of nanovoids. The initial $\mathrm{HgS}$ was monocrystalline $(0 \mathrm{~s})$. After the $50 \mathrm{~s}$ ebeam irradiation, the $\mathrm{Hg}$ nanodroplets with the diameter of $\sim 6 \mathrm{~nm}$ were observed (marked by the dotted circles). Such nanodroplets gradually shrank and finally vanished as the e-beam irradiation continued., Following the creation, growth and coalescence of nanovoids, the $\mathrm{HgS}$ nanobipyramid was gradually destroyed with the prolonged irradiation (120, 180 and 240 s). Eventually (300 s), a hollow nanostructure with the twisting shell layer was seen.

Figure 4. Size-dependent effect on the reaction dynamics of chiral HgS. Upper: Total reaction times of $\mathrm{HgS}$ nanobipyramids as a function of their sizes (length ranged from $\sim 80$ to $400 \mathrm{~nm}$ ) under the same e-beam excitation condition. The total reaction time firstly increased with the size, then reached the maximum at the size of $\sim 200 \mathrm{~nm}$ and after that decreased with increasing the crystal size. The inset illustrates the length of a nanobipyramid used for this work. Bottom: TEM images and corresponding FFT patterns of four typical HgS nanobipyramids with the different sizes ( 120, 200, 240 and $340 \mathrm{~nm})$, corresponding to the dotted circles marked in the top panel. These nanobipyramids were irradiated under the identical condition for $240 \mathrm{~s}$. The comparison of such structural evolution indicates the distinct irradiation stability of $\mathrm{HgS}$ with different sizes. 
Figure 5. Chiral effect on the reaction dynamics of HgS. Upper: TEM images and corresponding FFT patterns of HgS nanobipyramids ( $280 \mathrm{~nm})$ with chiral (left) and achiral (right) morphology under the $20 \mathrm{~s}$ e-beam irradiation. Several $\mathrm{Hg}$ nanodroplets with the diameter of 3-6 $\mathrm{nm}$ occurred in the achiral HgS (marked by the dotted circles), whereas no droplets were observed in the chiral one. Bottom: AFM topography images of $\mathrm{HgS}$ nanobipyramids with chiral (left) and achiral (right) morphology. The chiral surface was rougher than the achiral one.

Figure 6. Mechanism of the e-beam-induced chemical reactions of HgS. Upper: Schematic of the structural evolution of order-disorder transition upon the e-beam excitation. With the incoming highenergy electrons, the $\mathrm{Hg}-\mathrm{S}$ chemical bonds are cleaved, and the reduction of $\mathrm{Hg}^{2+}$ to $\mathrm{Hg}^{0}$ as well as the

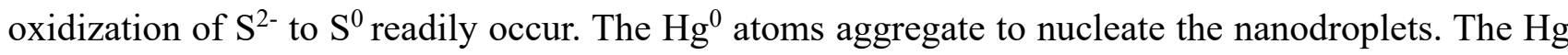
nanodroplets grow and evaporate, and subsequently a number of nanovoids arise, leading to the formation of many nano-sized crystals. Such small crystals gradually rotate and change their orientations, giving rise to the ring-patterned polycrystalline character. With the continuous irradiation, the nanostructures are severely disordered so that most parts of the sample finally become amorphous. Bottom left: The free energy of surface steps $\left(\Delta G_{s}\right)$, defects creation $\left(\Delta G_{d}\right)$ and launch of structural transition $\left(\Delta G_{t}\right)$ in the irradiated $\mathrm{HgS}$ nanobipyramid as a function of the crystal size $L$. Bottom middle: Free energy diagram for nucleation with respect to the radius of the nucleus. Bottom right: Evaporation time and the total free energy of the $\mathrm{Hg}$ nanodroplets as a function of the contact angle $\theta$. The inset shows the schematic of the $\mathrm{Hg}$ nanodroplet on a solid surface. 


\section{References}

1. Zhang, Y., Guo, J., Shi, L., Zhu, Y., Hou, K., Zheng, Y. \& Tang, Z. Tunable chiral metal organic frameworks toward visible light-driven asymmetric catalysis. Science advances. 3, e1701162 (2017).

2. Zhao, X., Zang, S. \& Chen, X. Stereospecific interactions between chiral inorganic nanomaterials and biological systems. Chem. Soc. Rev. 49, 2481-2503 (2020).

3. Kuzyk, A., Schreiber, R., Fan, Z., Pardatscher, G., Roller, E., Högele, A., Simmel, F. C., Govorov, A. O. \& Liedl, T. DNA-based self-assembly of chiral plasmonic nanostructures with tailored optical response. Nature. 483, 311-314 (2012).

4. Tsukube, H. \& Shinoda, S. Lanthanide complexes in molecular recognition and chirality sensing of biological substrates. Chem. Rev. 102, 2389-2404 (2002).

5. Dou, X., Mehwish, N., Zhao, C., Liu, J., Xing, C. \& Feng, C. Supramolecular Hydrogels with Tunable Chirality for Promising Biomedical Applications. Accounts Chem. Res. 53, 852-862 (2020).

6. Zhao, X., Zang, S. \& Chen, X. Stereospecific interactions between chiral inorganic nanomaterials and biological systems. Chem. Soc. Rev. 49, 2481-2503 (2020).

7. Cahn, R. S., Ingold, C. \& Prelog, V. Specification of molecular chirality. Angewandte Chemie International Edition in English. 5, 385-415 (1966).

8. Liu, M., Zhang, L. \& Wang, T. Supramolecular chirality in self-assembled systems. Chem. Rev. 115, 7304-7397 (2015).

9. Pályi, G., Zucchi, C. \& Caglioti, L. Progress in biological chirality. (Elsevier, 2004).

10. Yang, Y., Rice, B., Shi, X., Brandt, J. R., Correa Da Costa, R., Hedley, G. J., Smilgies, D., Frost, 
J. M., Samuel, I. D. \& Otero-De-La-Roza, A. Emergent properties of an organic semiconductor driven by its molecular chirality. Acs Nano. 11, 8329-8338 (2017).

11. Gao, X., Han, B., Yang, X. \& Tang, Z. Perspective of chiral colloidal semiconductor nanocrystals: opportunity and challenge. J. Am. Chem. Soc. 141, 13700-13707 (2019).

12. Purcell-Milton, F., McKenna, R., Brennan, L. J., Cullen, C. P., Guillemeney, L., Tepliakov, N. V., Baimuratov, A. S., Rukhlenko, I. D., Perova, T. S. \& Duesberg, G. S. Induction of chirality in two-dimensional nanomaterials: Chiral 2D MoS2 nanostructures. Acs Nano. 12, 954-964 (2018).

13. Hao, C., Xu, L., Sun, M., Ma, W., Kuang, H. \& Xu, C. Chirality on Hierarchical Self-Assembly of Au@AuAg Yolk-Shell Nanorods into Core-Satellite Superstructures for Biosensing in Human Cells. Adv. Funct. Mater. 28, 1802372 (2018).

14. Li, J., Li, J., Liu, R., Tu, Y., Li, Y., Cheng, J., He, T. \& Zhu, X. Autonomous discovery of optically active chiral inorganic perovskite nanocrystals through an intelligent cloud lab. Nat. Commun. 11, $1-10(2020)$.

15. Ben-Moshe, A., Maoz, B. M., Govorov, A. O. \& Markovich, G. Chirality and chiroptical effects in inorganic nanocrystal systems with plasmon and exciton resonances. Chem. Soc. Rev. 42, 70287041 (2013).

16. Xiao, L., An, T., Wang, L., Xu, X. \& Sun, H. Novel properties and applications of chiral inorganic nanostructures. Nano Today. 30, 100824 (2020).

17. Hentschel, M., Schäferling, M., Duan, X., Giessen, H. \& Liu, N. Chiral plasmonics. Science advances. 3, e1602735 (2017).

18. Hao, C., Gao, Y., Wu, D., Li, S., Xu, L., Wu, X., Guo, J., Sun, M., Li, X. \& Xu, C. Tailoring chiroptical activity of iron disulfide quantum dot hydrogels with circularly polarized light. Adv. 
Mater. 31, 1903200 (2019).

19. Hodgkinson, I. \& Wu, Q. H. Inorganic chiral optical materials. Adv. Mater. 13, 889-897 (2001).

20. Ma, L., Huang, Z., Duan, Y., Shen, X. \& Che, S. Optically active chiral Ag nanowires. Science China Materials. 58, 441-446 (2015).

21. Yan, J., Feng, W., Kim, J., Lu, J., Kumar, P., Mu, Z., Wu, X., Mao, X. \& Kotov, N. A. Selfassembly of chiral nanoparticles into semiconductor helices with tunable near-infrared optical activity. Chem. Mater. 32, 476-488 (2019).

22. Shindo, H., Shirota, Y., Niki, K., Kawasaki, T., Suzuki, K., Araki, Y., Matsumoto, A. \& Soai, K. Asymmetric autocatalysis induced by cinnabar: observation of the enantioselective adsorption of a 5-pyrimidyl alkanol on the crystal surface. Angewandte Chemie. 125, 9305-9308 (2013).

23. Soai, K., Osanai, S., Kadowaki, K., Yonekubo, S., Shibata, T. \& Sato, I. D-and L-quartz-promoted highly enantioselective synthesis of a chiral organic compound. J. Am. Chem. Soc. 121, 1123511236 (1999).

24. Mallat, T., Orglmeister, E. \& Baiker, A. Asymmetric catalysis at chiral metal surfaces. Chem. Rev. 107, 4863-4890 (2007).

25. Li, S., Liu, J., Ramesar, N. S., Heinz, H., Xu, L., Xu, C. \& Kotov, N. A. Single-and multicomponent chiral supraparticles as modular enantioselective catalysts. Nat. Commun. 10, 1-10 (2019).

26. Flack, H. D. Chiral and achiral crystal structures. Helv. Chim. Acta. 86, 905-921 (2003).

27. Dryzun, C. \& Avnir, D. On the abundance of chiral crystals. Chem. Commun. 48, 5874-5876 (2012).

28. Fan, J. \& Kotov, N. A. Chiral Nanoceramics. Adv. Mater., 1906738 (2020). 
29. Fan, Z. \& Govorov, A. O. Chiral nanocrystals: plasmonic spectra and circular dichroism. Nano Lett. 12, 3283-3289 (2012).

30. Hananel, U., Ben-Moshe, A., Diamant, H. \& Markovich, G. Spontaneous and directed symmetry breaking in the formation of chiral nanocrystals. Proceedings of the National Academy of Sciences. 116, 11159-11164 (2019).

31. González-Rubio, G., Mosquera, J., Kumar, V., Pedrazo-Tardajos, A., Llombart, P., Solís, D. M., Lobato, I., Noya, E. G., Guerrero-Martínez, A. \& Taboada, J. M. Micelle-directed chiral seeded growth on anisotropic gold nanocrystals. Science. 368, 1472-1477 (2020).

32. Lee, H., Ahn, H., Mun, J., Lee, Y. Y., Kim, M., Cho, N. H., Chang, K., Kim, W. S., Rho, J. \& Nam, K. T. Amino-acid-and peptide-directed synthesis of chiral plasmonic gold nanoparticles. Nature. 556, 360-365 (2018).

33. Tian, F. \& Chen, R. Pd-Mediated Synthesis of Ag33 Chiral Nanocluster with Core-Shell Structure in T Point Group. J. Am. Chem. Soc. 141, 7107-7114 (2019).

34. Wang, P., Yu, S., Govorov, A. O. \& Ouyang, M. Cooperative expression of atomic chirality in inorganic nanostructures. Nat. Commun. 8, 1-9 (2017).

35. Lv, J., Ding, D., Yang, X., Hou, K., Miao, X., Wang, D., Kou, B., Huang, L. \& Tang, Z. Biomimetic chiral photonic crystals. Angewandte Chemie. 131, 7865-7869 (2019).

36. Kuno, J., Miyake, K., Katao, S., Kawai, T. \& Nakashima, T. Enhanced Enantioselectivity in the Synthesis of Mercury Sulfide Nanoparticles through Ostwald Ripening. Chem. Mater., (2020).

37. Oujja, M., Sanz, M., Rebollar, E., Marco, J. F., Domingo, C., Pouli, P., Kogou, S., Fotakis, C. \& Castillejo, M. Wavelength and pulse duration effects on laser induced changes on raw pigments used in paintings. Spectrochimica Acta Part A: Molecular and Biomolecular Spectroscopy. 102, 
7-14 (2013).

38. Yu, J., Warren, W. S. \& Fischer, M. C. Visualization of vermilion degradation using pump-probe microscopy. Science advances. 5, w3136 (2019).

39. Krasheninnikov, A. V., Banhart, F., Li, J. X., Foster, A. S. \& Nieminen, R. M. Stability of carbon nanotubes under electron irradiation: role of tube diameter and chirality. Physical review B. 72, $125428(2005)$.

40. Celik-Aktas, A., Zuo, J. M., Stubbins, J. F., Tang, C. \& Bando, Y. Structure and chirality distribution of multiwalled boron nitride nanotubes. Appl. Phys. Lett. 86, 133110 (2005).

41. Momma, K. \& Izumi, F. VESTA 3 for three-dimensional visualization of crystal, volumetric and morphology data. Journal of applied crystallography. 44, 1272-1276 (2011).

42. Nusimovici, M. A. \& Gorre, G. Phonons in cinnabar. Physical Review B. 8, 1648 (1973).

43. Ballirano, P., Botticelli, M. \& Maras, A. Thermal behaviour of cinnabar, $\alpha-\mathrm{HgS}$, and the kinetics of the $\beta-\mathrm{HgS}$ (metacinnabar) $\rightarrow \alpha-\mathrm{HgS}$ conversion at room temperature. Eur. J. Mineral. 25, 957965 (2013).

44. He, J., Liu, Z., Cao, Z., Zhang, H., Meng, Y., Chen, B. \& Zhong, D. Visualizing the Redox Reaction Dynamics of Perovskite Nanocrystals in Real and Reciprocal Space. The Journal of Physical Chemistry Letters. 11, 2550-2558 (2020).

45. Lv, J., Yang, J., Li, X. \& Chai, Z. Size dependent radiation-stability of $\mathrm{ZnO}$ and $\mathrm{TiO}_{2}$ particles. Dyes Pigments. 164, 87-90 (2019).

46. Barr, C. M., Li, N., Boyce, B. L. \& Hattar, K. Examining the influence of grain size on radiation tolerance in the nanocrystalline regime. Appl. Phys. Lett. 112, 181903 (2018).

47. Zhang, X., Hattar, K., Chen, Y., Shao, L., Li, J., Sun, C., Yu, K., Li, N., Taheri, M. L. \& Wang, 
H. Radiation damage in nanostructured materials. Prog. Mater. Sci. 96, 217-321 (2018).

48. Andrievski, R. A. Behavior of radiation defects in nanomaterials. Rev. Adv. Mater. Sci. 29, 54-67 (2011).

49. Malac, M., Schoefield, M., Zhu, Y. \& Egerton, R. Exposure characteristics of cobalt fluoride $\left(\mathrm{CoF}_{2}\right)$ self-developing electron-beam resist on sub-100 nm scale. J. Appl. Phys. 92, 1112-1121 (2002).

50. Hultgren, R. R. \& Orr, R. L. Selected values of thermodynamic properties of metals and alloys. (John Wiley \& Sons Inc, 1963).

51. Hepler, L. G. \& Olofsson, G. Mercury. Thermodynamic properties, chemical equilibriums, and standard potentials. Chem. Rev. 75, 585-602 (1975).

52. Wang, S., Javadpour, F. \& Feng, Q. Confinement correction to mercury intrusion capillary pressure of shale nanopores. Sci. Rep.-Uk. 6, 20160 (2016).

53. Erbil, H. Y. Evaporation of pure liquid sessile and spherical suspended drops: A review. $A d v$. Colloid Interfac. 170, 67-86 (2012).

54. Lugg, G. A. Diffusion coefficients of some organic and other vapors in air. Anal. Chem. 40, 10721077 (1968).

55. Bennett, T. D. \& Farrelly, M. Vaporization kinetics during pulsed laser heating of liquid Hg. J. Heat Transfer. 122, 345-350 (2000).

56. Suh, D. \& Yasuoka, K. Nanoscale droplet vaporisation by molecular dynamics. Mol. Simulat. 41, 896-904 (2015).

57. Walther, J. H. \& Koumoutsakos, P. Molecular Dynamics Simulation of Nanodroplet Evaporation. Journal of Heat Transfer. 123, 741-748 (2000). 
58. Varanasi, K. K., Hsu, M., Bhate, N., Yang, W. \& Deng, T. Spatial control in the heterogeneous nucleation of water. Appl. Phys. Lett. 95, 94101 (2009). 


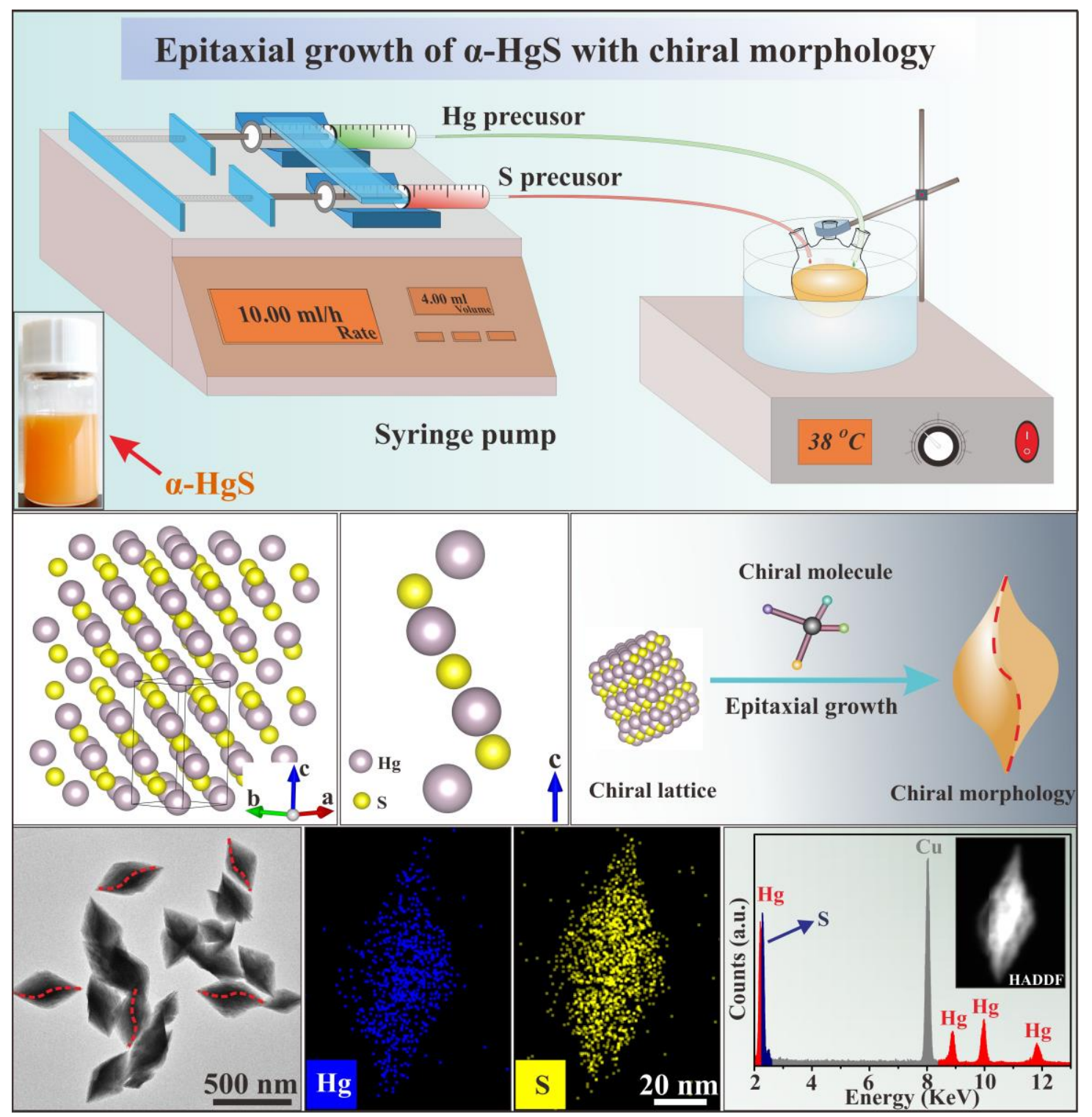

Figure 1 


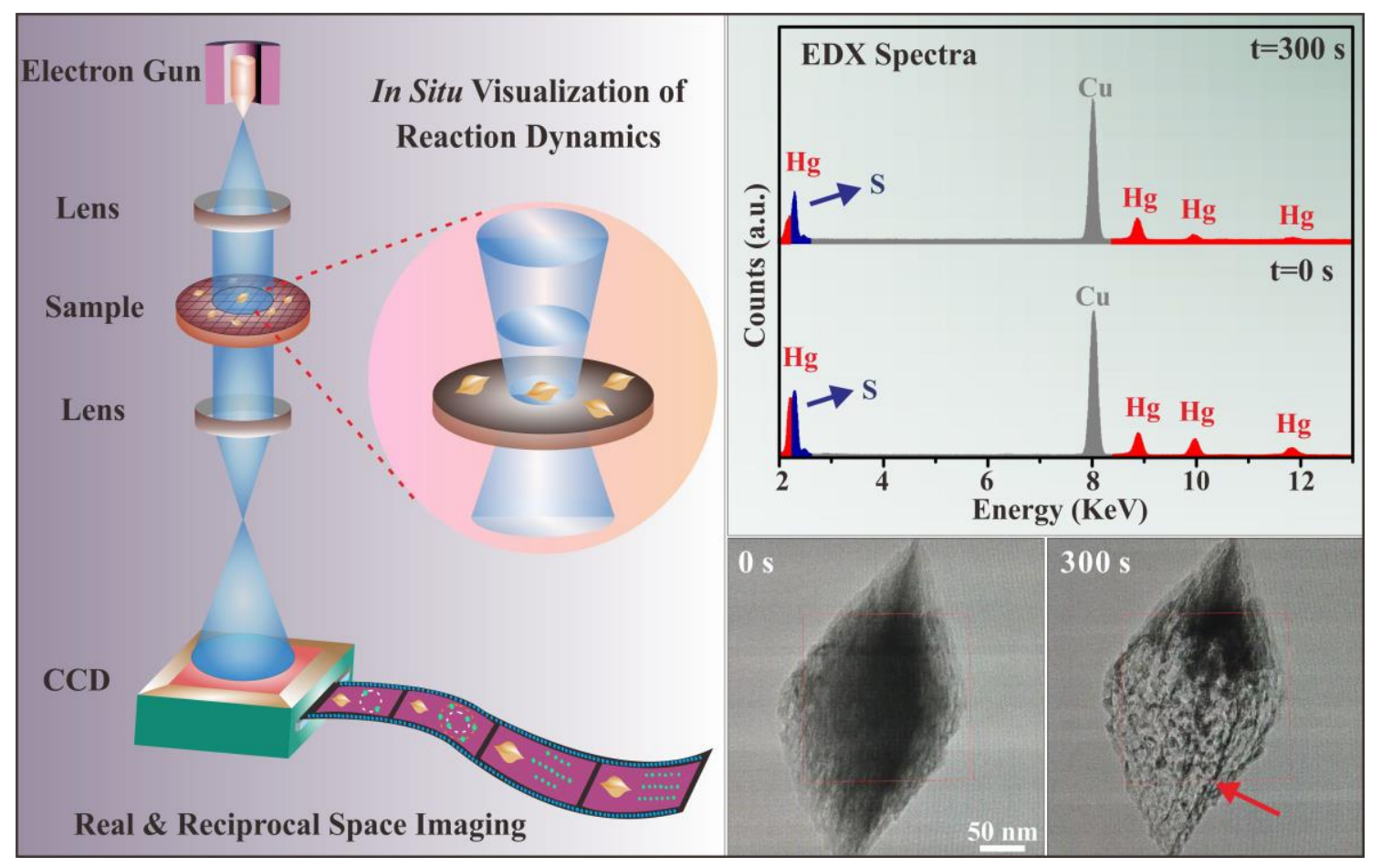

Figure 2 


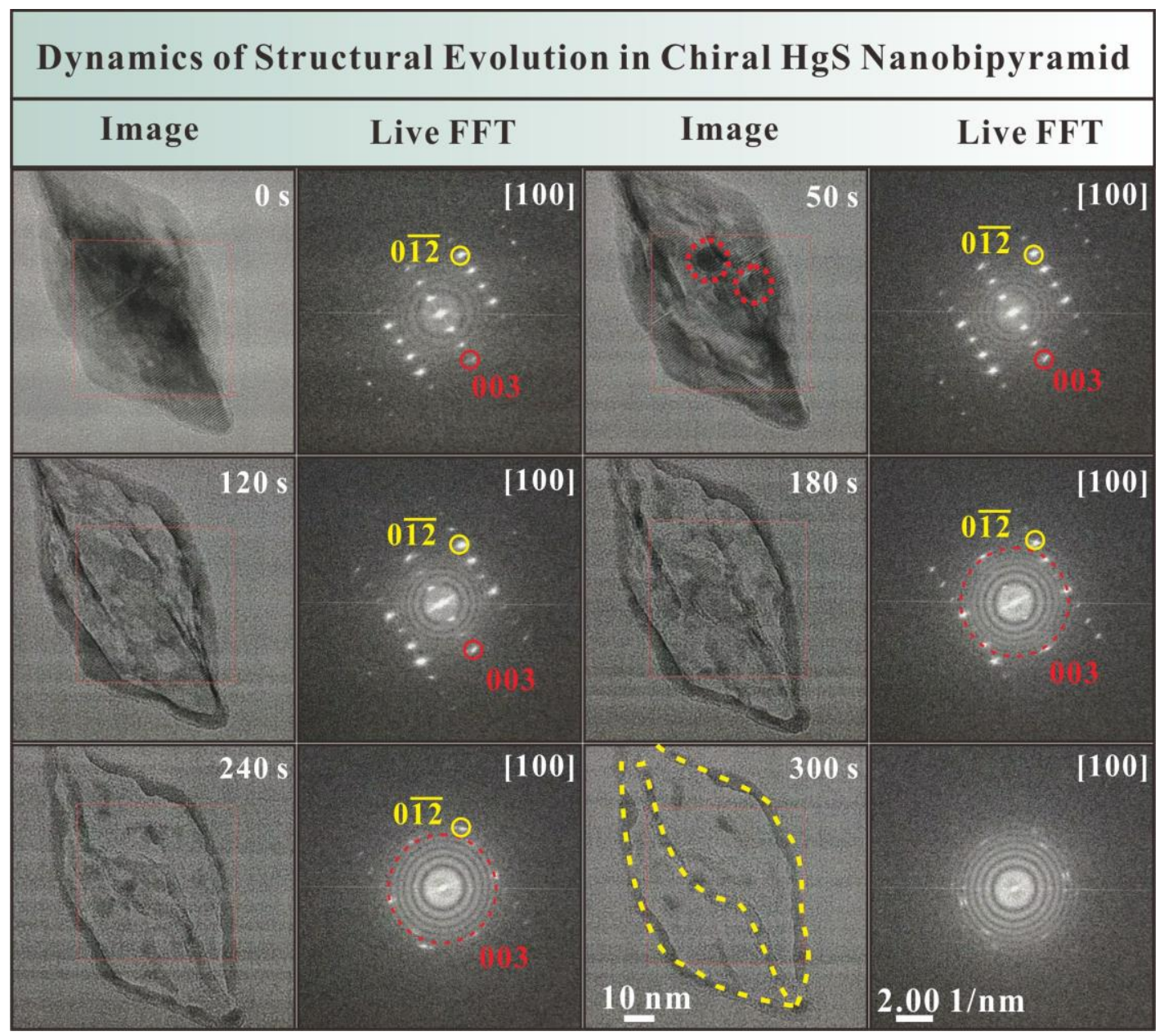

Figure 3 


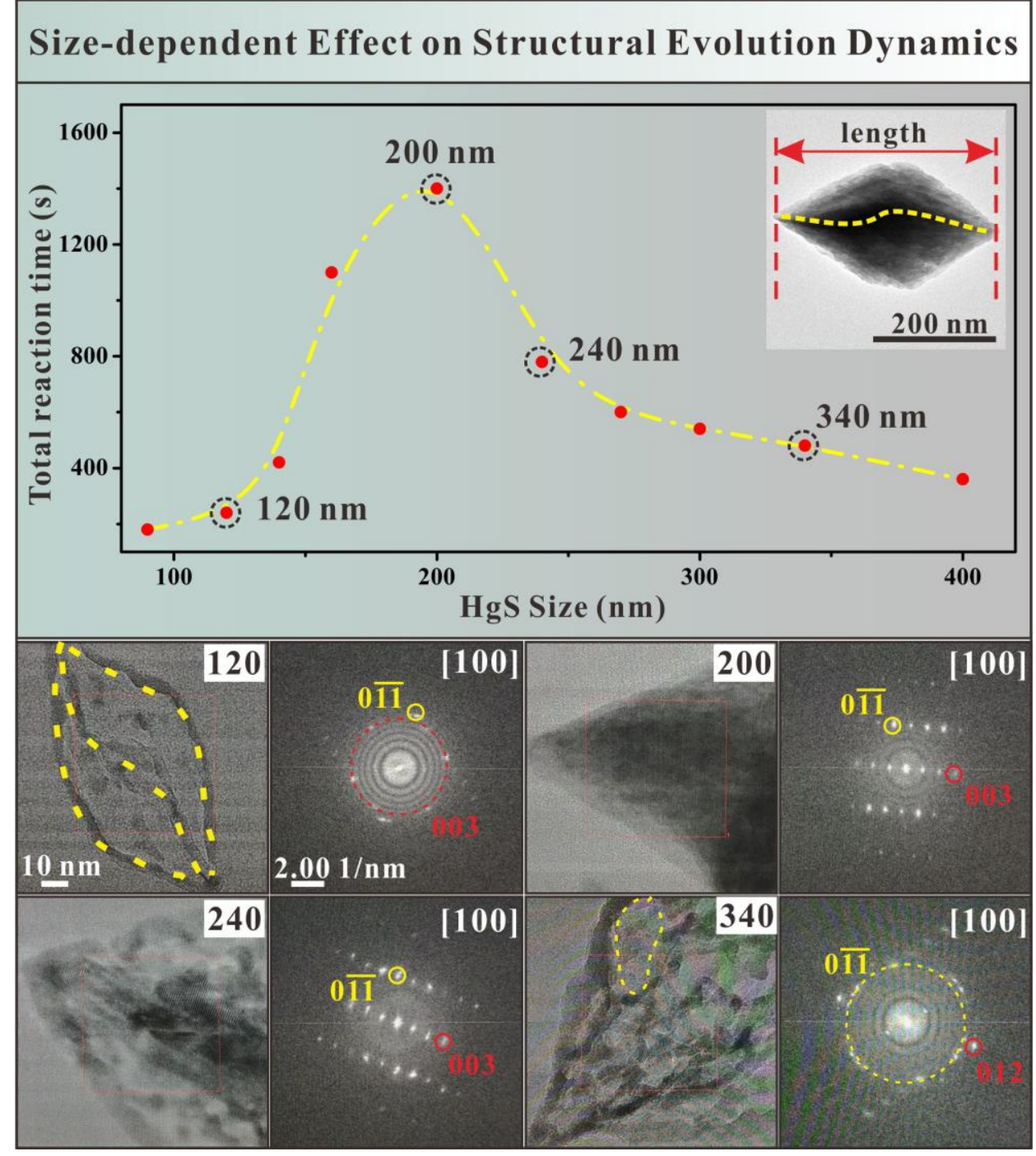

Figure 4 


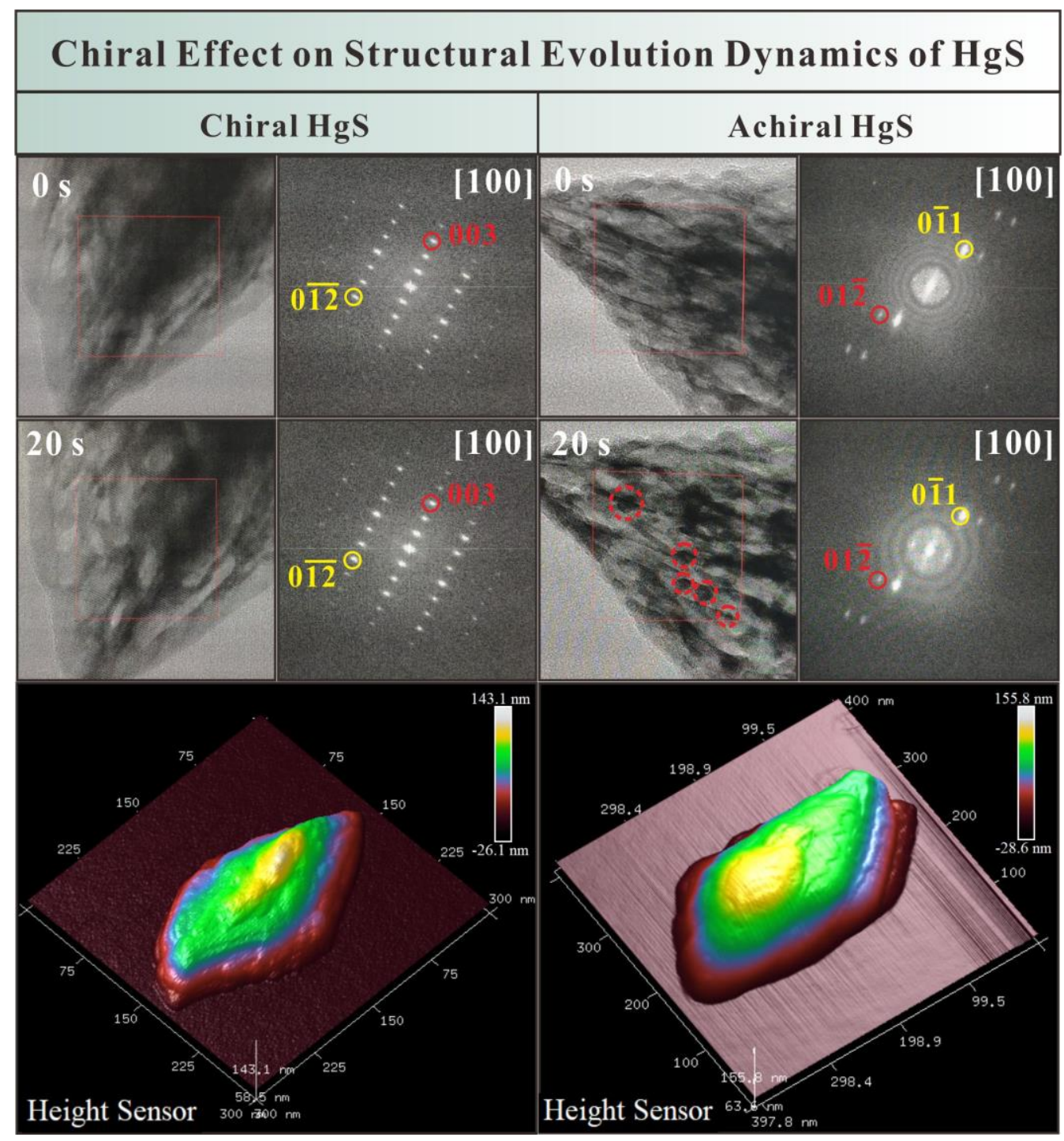

Figure 5 


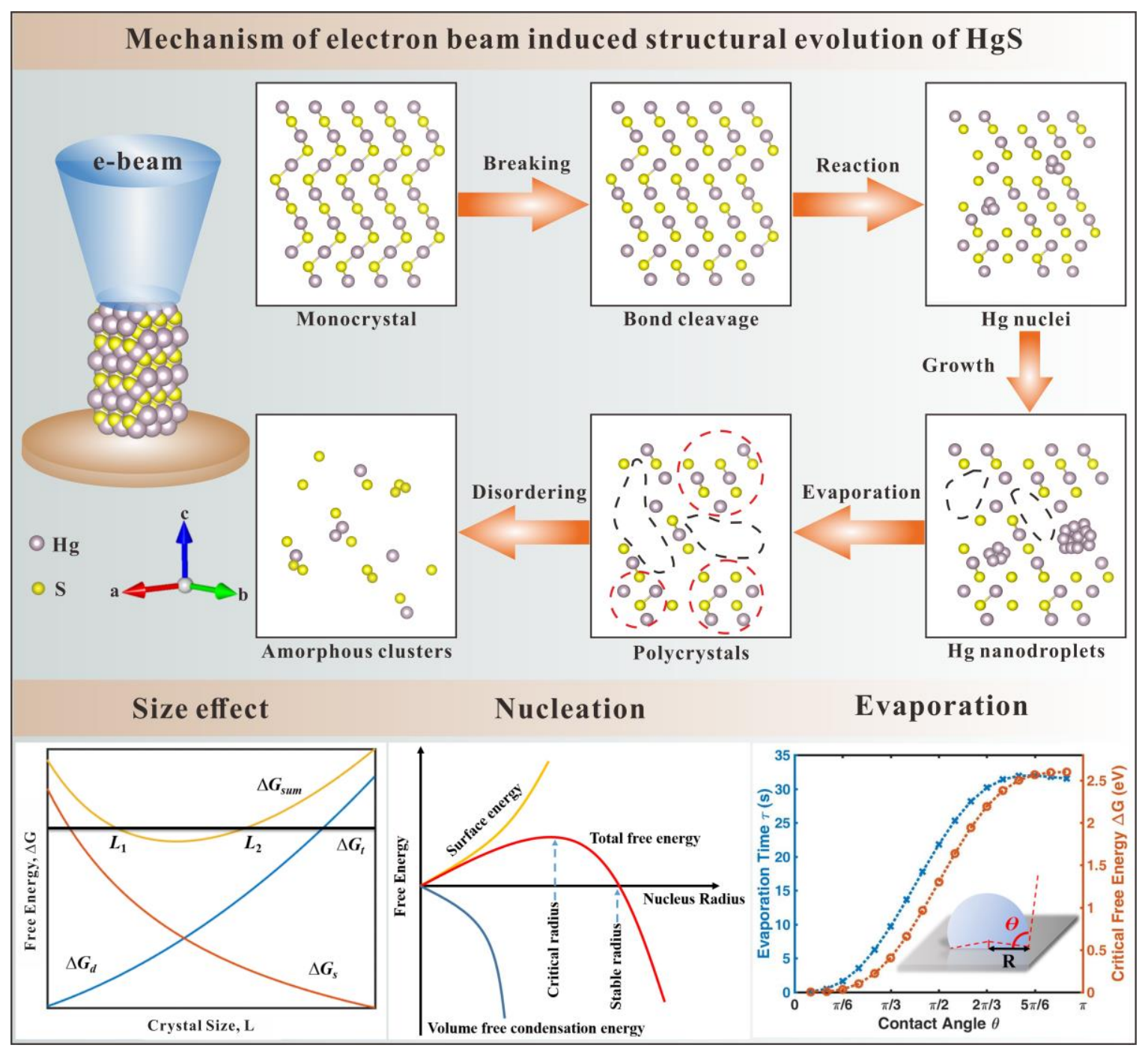

Figure 6 


\section{Figures}

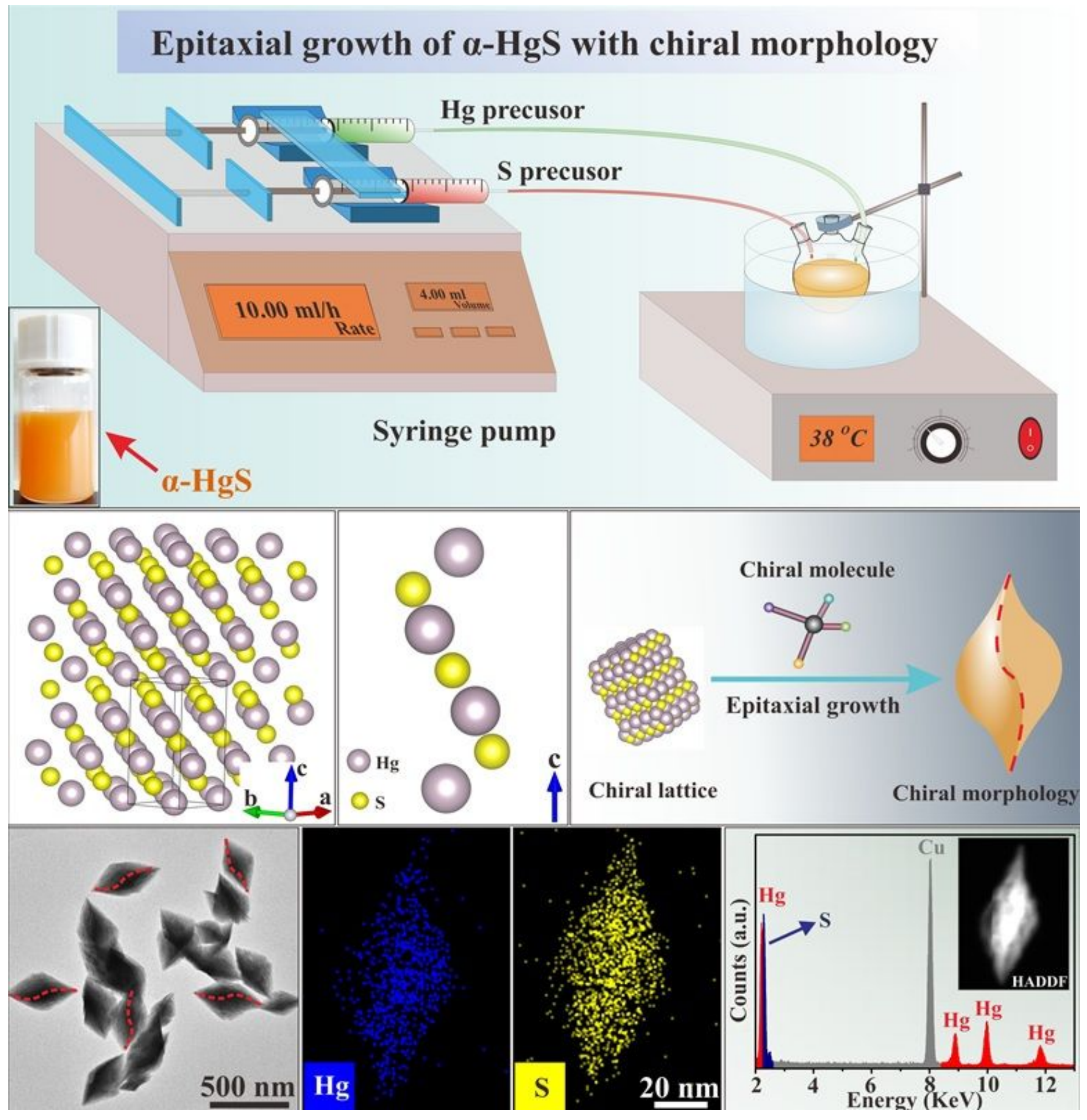

Figure 1

Synthesis and structural characterization of a-HgS nanobipyramids. Upper: Schematic of the seedmediated epitaxial growth of chiral a-HgS nanostructures. The inset shows the orange a-HgS colloidal suspensions. Middle left: Schematic representation of the atomic structure of a-HgS (Space group 
P3121). Middle center: Helical chains of $\mathrm{Hg}$ and $\mathrm{S}$ atoms along the crystallographic c axis. Middle right: Schematic illustration of the epitaxial evolution from the chiral lattice to the chiral morphology by involving the chiral molecules. Bottom left: Typical TEM image of a-HgS with chiral morphology. The dotted lines are the twisting orientations in the nanobipyramids. Bottom center: Elemental mapping of $\mathrm{Hg}$ and S, respectively. Bottom right: EDX spectrum of of a-HgS. The inset is the corresponding high-angle annular dark field (HAADF) image for EDX.
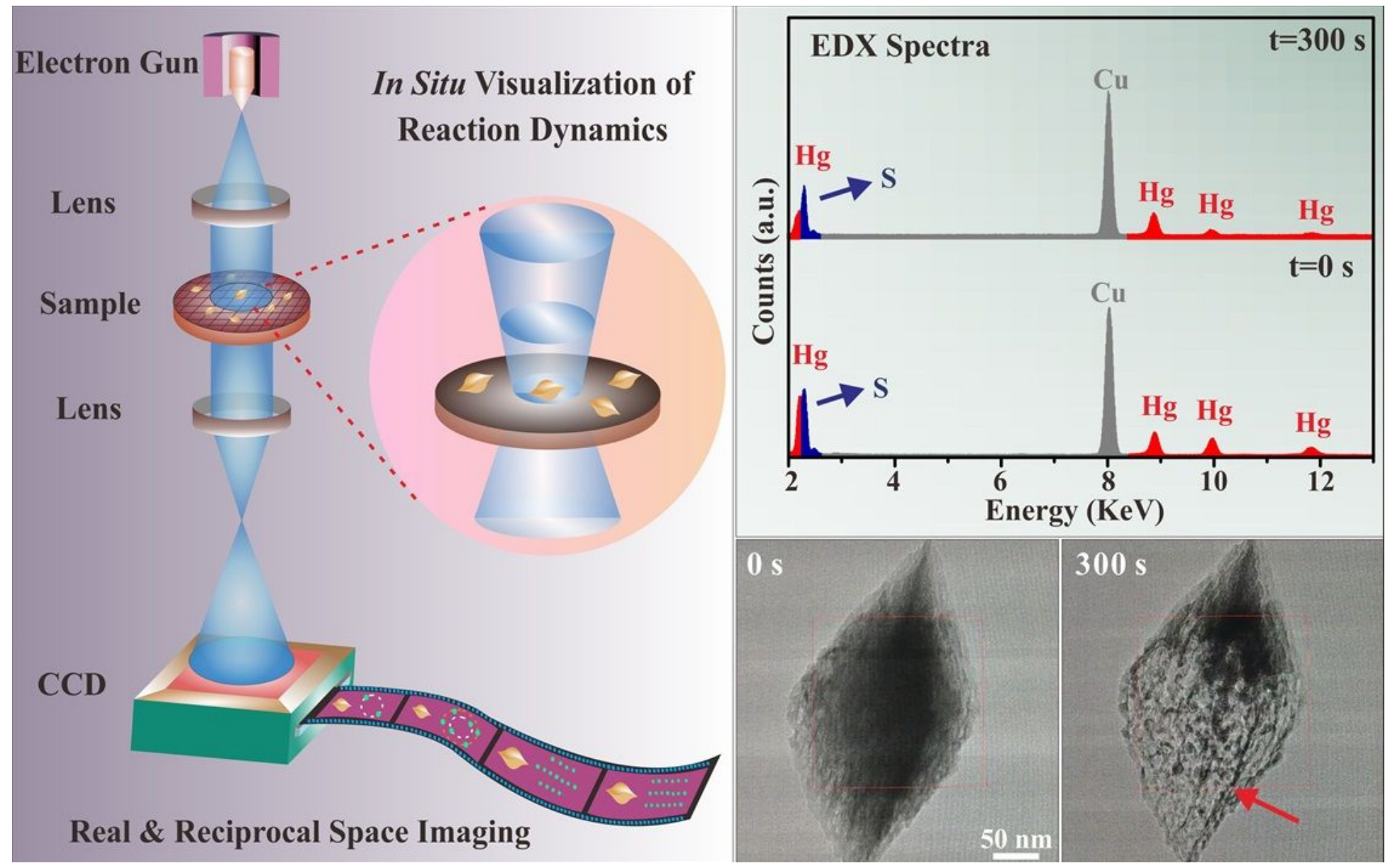

\section{Figure 2}

Experimental setup for in-situ observations of chemical reaction dynamics. Left: Schematic of e-beaminduced chemical reaction dynamics visualized in both real and reciprocal space. The sample dynamics are triggered by an e-beam, while their structural evolution and corresponding diffraction patterns are simultaneously recorded by a charged-coupled device (CCD) camera. Upper right: EDX spectra of a-HgS before and after the $300 \mathrm{~s}$ e-beam irradiation. Bottom right: TEM images of a-HgS before and after the $300 \mathrm{~s}$ e-beam irradiation. The arrow indicates the occurrence of nanoclusters due to the irradiation. 


\section{Dynamics of Structural Evolution in Chiral HgS Nanobipyramid}

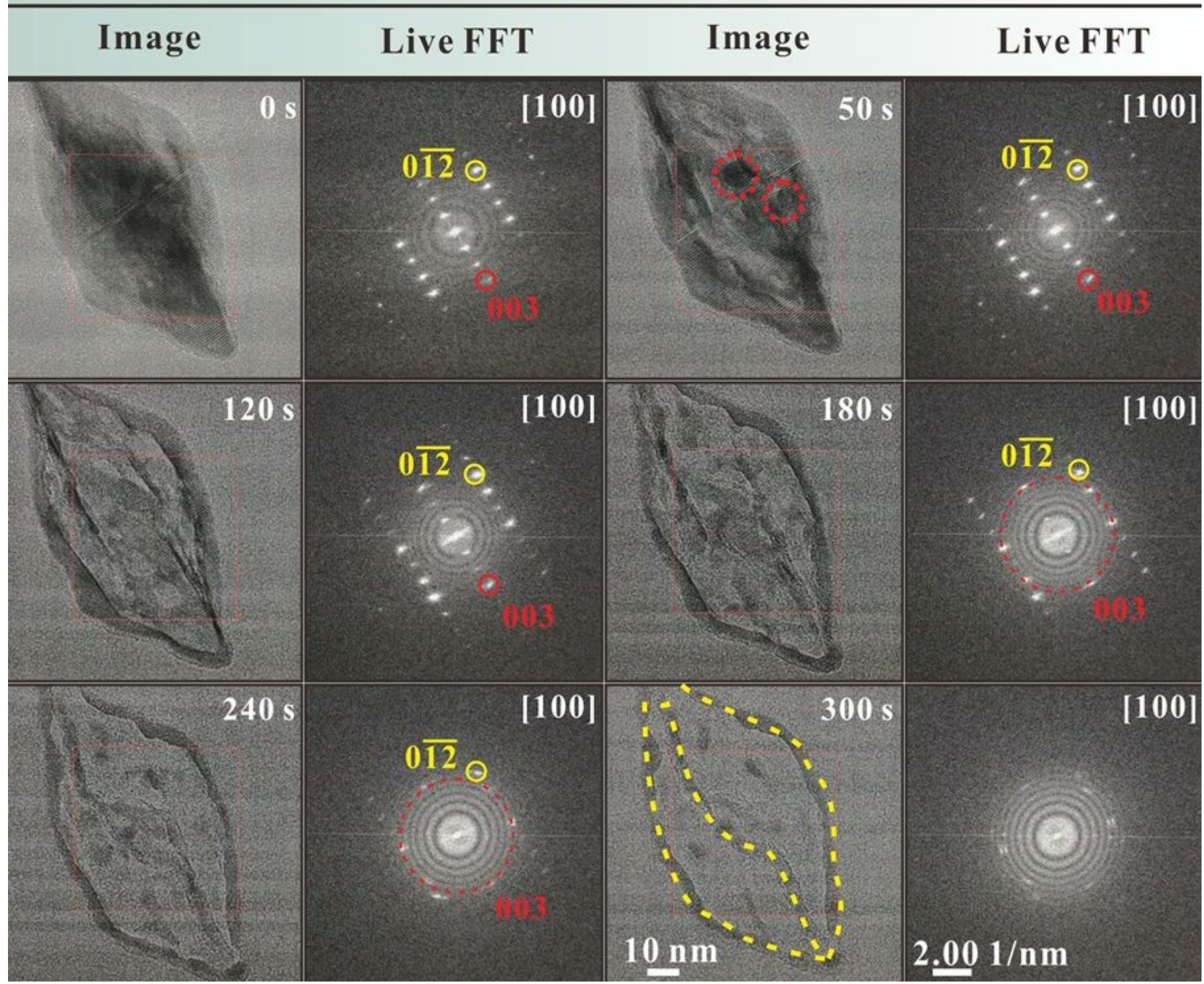

\section{Figure 3}

Structural evolution in the chiral a-HgS nanobipyramid. Time-resolved TEM images and live FFT patterns show the formation and evaporation dynamics of $\mathrm{Hg}$ nanodroplets as well as the creation and coalescence of nanovoids. The initial HgS was monocrystalline ( $0 \mathrm{~s})$. After the $50 \mathrm{~s}$ e-beam irradiation, the $\mathrm{Hg}$ nanodroplets with the diameter of $\sim 6 \mathrm{~nm}$ were observed (marked by the dotted circles). Such nanodroplets gradually shrank and finally vanished as the e-beam irradiation continued., Following the creation, growth and coalescence of nanovoids, the HgS nanobipyramid was gradually destroyed with the prolonged irradiation (120, 180 and 240 s). Eventually (300 s), a hollow nanostructure with the twisting shell layer was seen. 


\section{Size-dependent Effect on Structural Evolution Dynamics}

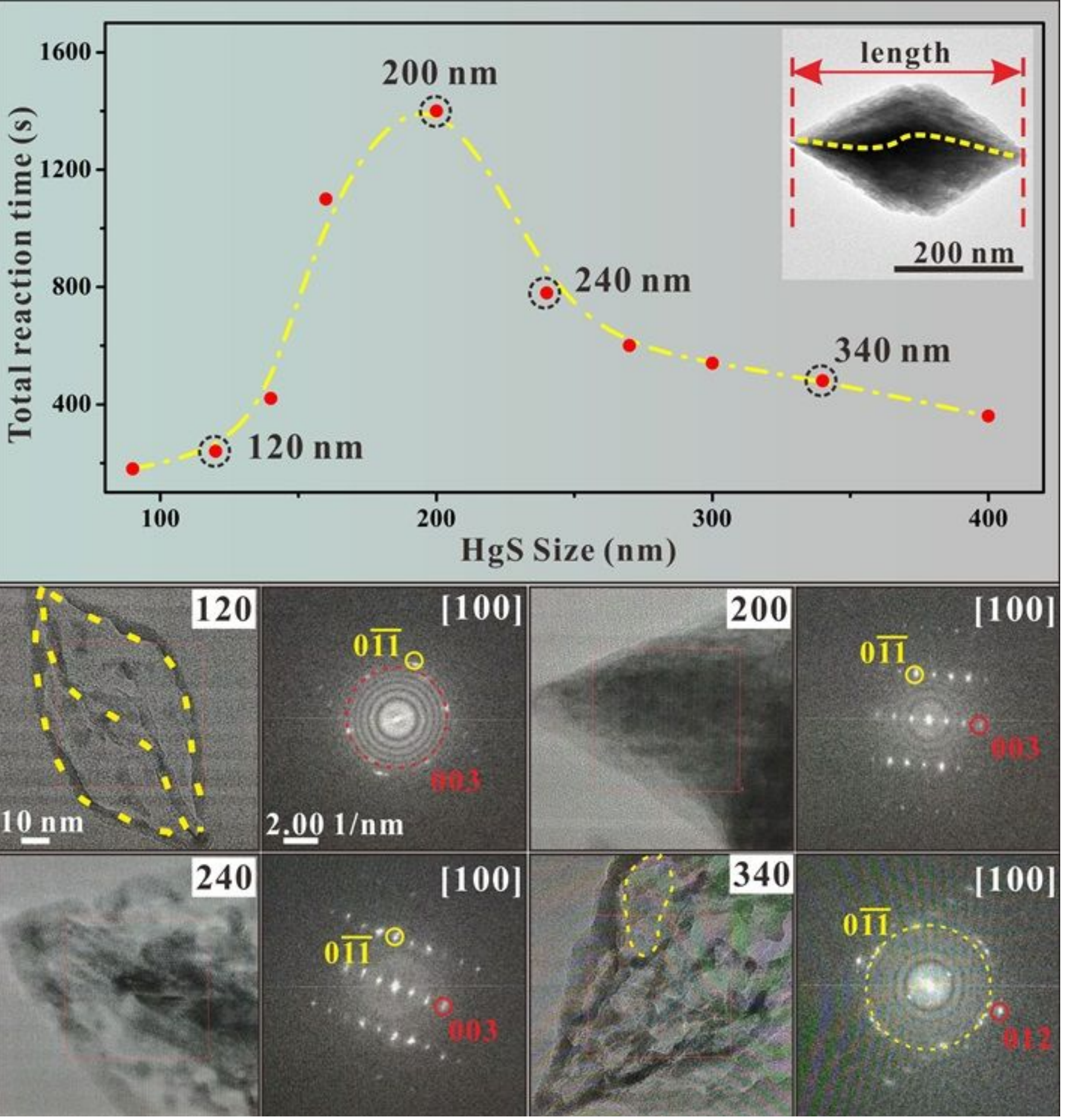

\section{Figure 4}

Size-dependent effect on the reaction dynamics of chiral HgS. Upper: Total reaction times of $\mathrm{HgS}$ nanobipyramids as a function of their sizes (length ranged from $~ 80$ to $400 \mathrm{~nm}$ ) under the same e-beam excitation condition. The total reaction time firstly increased with the size, then reached the maximum at the size of $\sim 200 \mathrm{~nm}$ and after that decreased with increasing the crystal size. The inset illustrates the length of a nanobipyramid used for this work. Bottom: TEM images and corresponding FFT patterns of 
four typical HgS nanobipyramids with the different sizes ( 120, 200, 240 and $340 \mathrm{~nm})$, corresponding to the dotted circles marked in the top panel. These nanobipyramids were irradiated under the identical condition for $240 \mathrm{~s}$. The comparison of such structural evolution indicates the distinct irradiation stability of HgS with different sizes.

\section{Chiral Effect on Structural Evolution Dynamics of HgS}

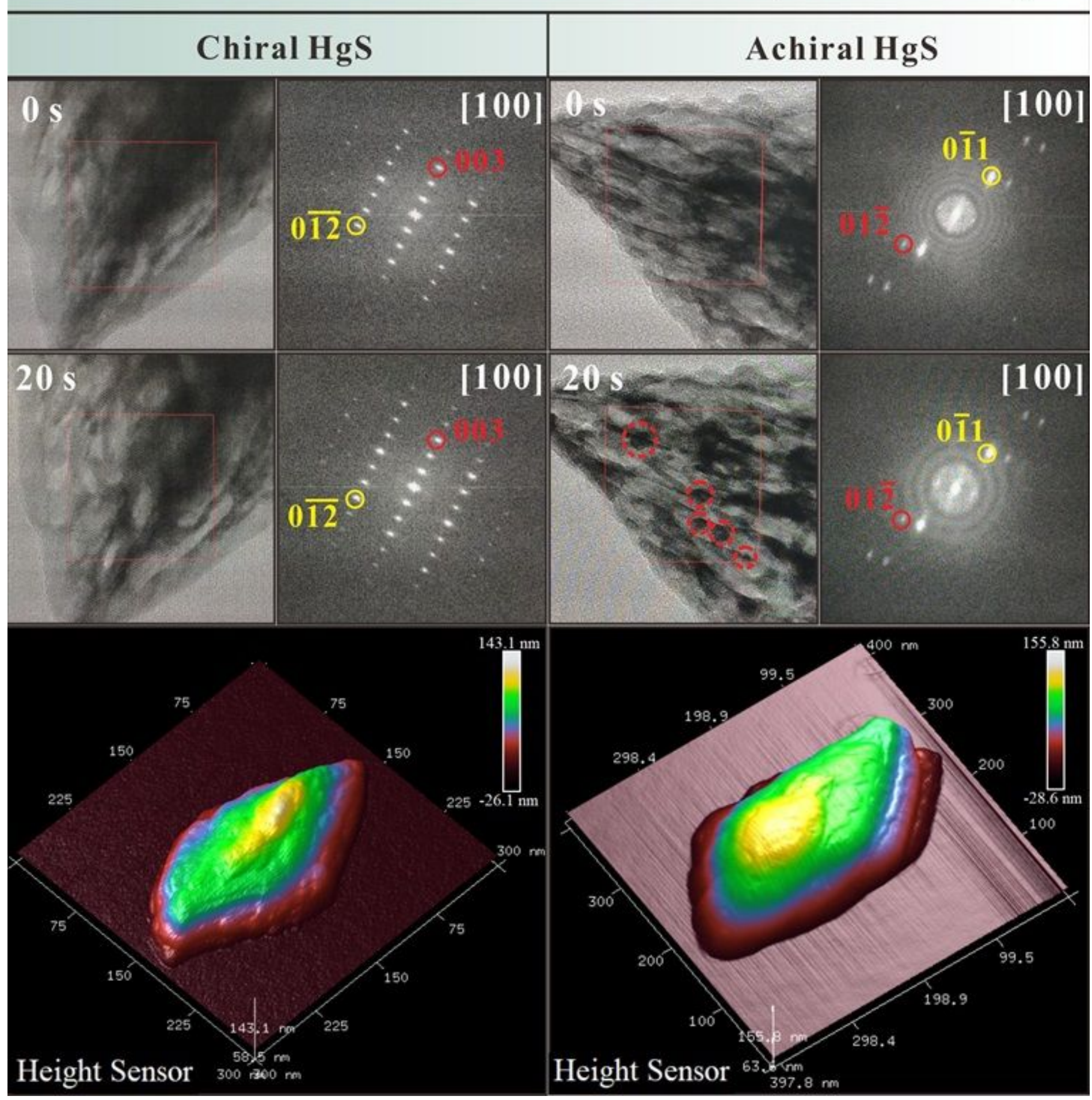

Figure 5 
Chiral effect on the reaction dynamics of HgS. Upper: TEM images and corresponding FFT patterns of HgS nanobipyramids ( $280 \mathrm{~nm}$ ) with chiral (left) and achiral (right) morphology under the $20 \mathrm{~s}$ e-beam irradiation. Several $\mathrm{Hg}$ nanodroplets with the diameter of 3-6 $\mathrm{nm}$ occurred in the achiral HgS (marked by the dotted circles), whereas no droplets were observed in the chiral one. Bottom: AFM topography images of HgS nanobipyramids with chiral (left) and achiral (right) morphology. The chiral surface was rougher than the achiral one.

\section{Mechanism of electron beam induced structural evolution of $\mathrm{HgS}$}

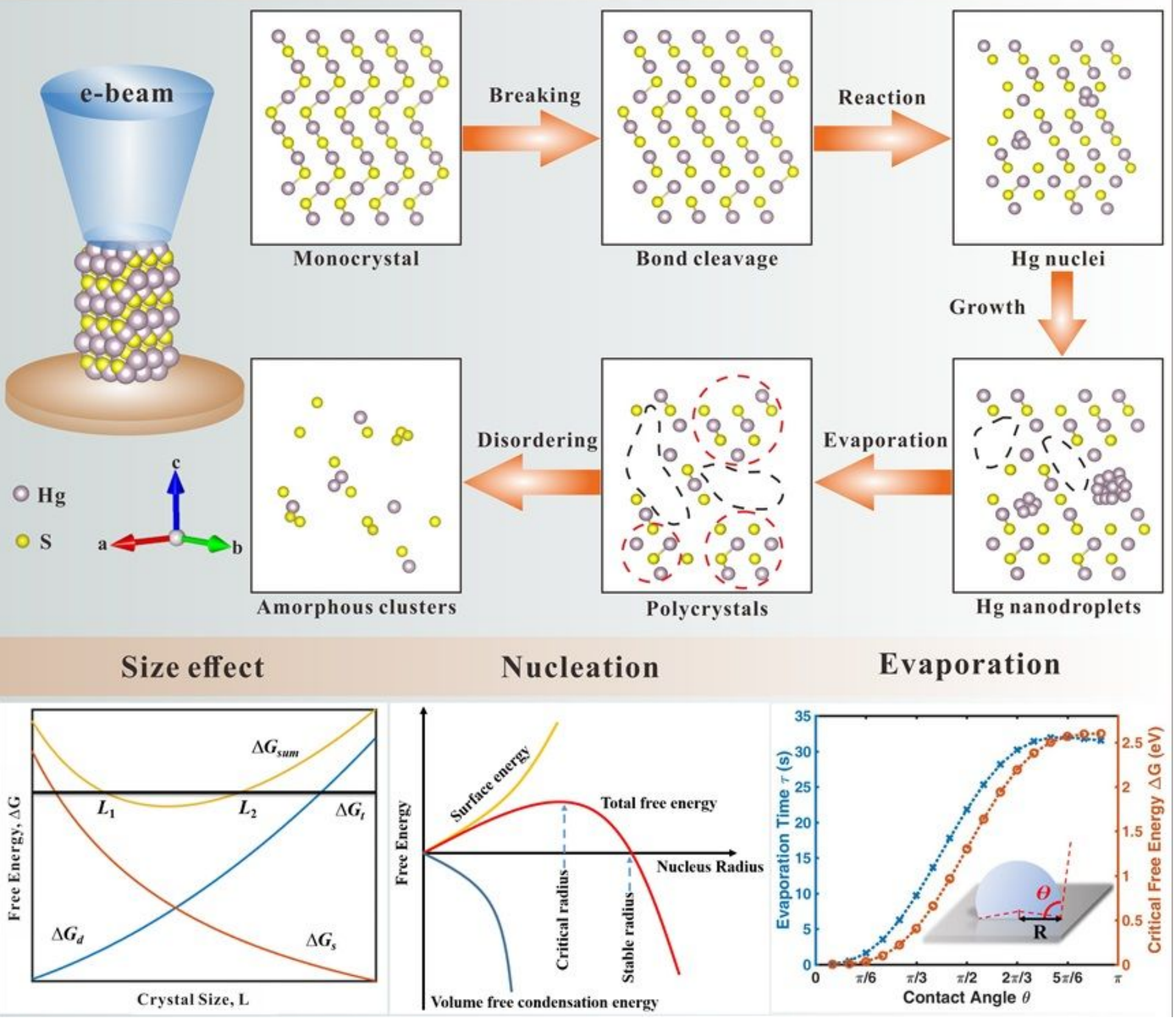

Figure 6

Mechanism of the e-beam-induced chemical reactions of HgS. Upper: Schematic of the structural evolution of order-disorder transition upon the e-beam excitation. With the incoming high-energy electrons, the $\mathrm{Hg}-\mathrm{S}$ chemical bonds are cleaved, and the reduction of $\mathrm{Hg} 2+$ to $\mathrm{HgO}$ as well as the 
oxidization of S2- to S0 readily occur. The $\mathrm{HgO}$ atoms aggregate to nucleate the nanodroplets. The $\mathrm{Hg}$ nanodroplets grow and evaporate, and subsequently a number of nanovoids arise, leading to the formation of many nano-sized crystals. Such small crystals gradually rotate and change their orientations, giving rise to the ring-patterned polycrystalline character. With the continuous irradiation, the nanostructures are severely disordered so that most parts of the sample finally become amorphous. Bottom left: The free energy of surface steps $\left(\Delta G_{-} s\right)$, defects creation $\left(\Delta G_{-} d\right)$ and launch of structural transition $\left(\Delta G_{-} t\right)$ in the irradiated $\mathrm{HgS}$ nanobipyramid as a function of the crystal size $\mathrm{L}$. Bottom middle: Free energy diagram for nucleation with respect to the radius of the nucleus. Bottom right: Evaporation time and the total free energy of the $\mathrm{Hg}$ nanodroplets as a function of the contact angle $\theta$. The inset shows the schematic of the Hg nanodroplet on a solid surface.

\section{Supplementary Files}

This is a list of supplementary files associated with this preprint. Click to download.

- Movie1.mp4

- Movie2.mp4

- SupplementaryMaterials.docx 